\title{
¿Cómo perciben los entrenadores de fútbol el rol del psicólogo del deporte?: un estudio comparativo
}

\author{
How perceived the soccer coaches the role of sport \\ psychologist: a comparative study
}

\section{Como percebem os treinadores de futebol o papel do psicólogo do desporto? : um estudo comparativo}

\author{
R. De la Vega Marcos ${ }^{1}$, M. Román Tabanera ${ }^{2}$, R. Ruiz Barquín ${ }^{1}$ y J.M. Hernández López
}

1 Universidad Autónoma de Madrid, 2 Máster en Psicología del Deporte (UAM-COE)

Resumen: Pese al reconocimiento público y científico del papel que desempeñan las diferentes variables psicológicas en el rendimiento deportivo, existen pocos datos que permitan evidenciar cuál es la percepción que tienen los máximos responsables de la coordinación del trabajo y del entrenamiento deportivo, es decir, los entrenadores, sobre el rol que desempeńa el psicólogo del deporte en un deporte tan extendido como es el fútbol. En el presente trabajo, se ha diseñado el Cuestionario de Evaluación de las Ideas Previas de los Entrenadores de Fútbol, aplicándolo a dos muestras de un total de 198 entrenadores de fútbol, durante las temporadas 2003 y 2011. La comparación de los resultados, permite evidenciar cierto patrón de estabilidad en los resultados, si bien se profundiza en las diferencias encontradas, así como en la importancia de superar las barreras manifestadas por los técnicos.

Palabras clave: Psicólogo del deporte, ideas previas, competencia profesional, formación.

Abstract: Despite the scientific and public recognition of the role of psychological variables in athletic performance, there is little evidence to show what is the coaches' perception on the role played by sport psychologist in a widespread sport like football. In this paper, we have designed the Cuestionario de Evaluación de las Ideas Previas de los Entrenadores de Fútbol, applying it to two samples of 198 football coaches during the 2003 and
2011 seasons. The comparison of results makes evident certain pattern of stability in the results, although the differences in depth, as well as the importance of overcoming the barriers raised by the coaches.

Keywords: Sport psychologist, Preconceptions, Professional competence, Training.

Resumo: Pese o reconhecimento público e científico do papel que desempenham as diferentes variáveis psicológicas no rendimento desportivo, existem pouco dados que permitam evidenciar qual é a percepção que têm os responsáveis máximos da coordenação do trabalho e do treino desportivo, isto é, os treinadores, sobre o papel que desempenha o psicólogo do desporto num desporto tấo divulgado com é o futebol. No presente trabalho, foi criado o Cuestionario de Evaluación de las Ideas Previas de los Entrenadores de Fútbol, sendo este aplicado a duas amostras de um total de 198 treinadores de futebol, durante as temporadas 2003 e 2011. A comparaçáo dos resultados permite evidenciar um certo padrão de estabilidade nos resultados, sendo aprofundadas as diferenças encontradas, assim como a importância de superar as barreiras manifestadas pelos técnicos.

Palavras-chave: Psicólogo do desporto, ideias prévias, competência profissional, formação.

\section{Introducción}

Demostrar la utilidad del entrenamiento de habilidades psicológicas en el ámbito deportivo ha sido y es uno de los objetivos fundamentales dentro de la Psicología del Deporte. Las investigaciones en este campo llevan años mostrando que la aplicación de programas psicológicos para el entrenamiento de habilidades como el control de estrés, la programación de objetivos o el control de la atención, mejoran el rendimiento de los deportistas (Gucciardi, 2012; Sousa, Cruz, Torregrosa, Vilches

\footnotetext{
Dirección para correspondencia [Correspodence address]: Ricardo De la Vega-Marcos. Universidad Autónoma de Madrid. Facultad de Formación de Profesorado y Educación. Departamento de Educación Física, Deporte y Motricidad humana. Despacho: III-314. Campus de Cantoblanco C/ Francisco Tomas y Valiente, 328049 Madrid. TF: 914973057. E-mail: ricardo.delavega@uam.es
}

\& Viladrich, 2006). Mientras que este interés ha partido, tradicionalmente, de los propios psicólogos del deporte, existen muy pocos estudios que permitan analizar la percepción que los técnicos tienen acerca de este rol profesional, sus posibles aportaciones y las barreras principales con las que se puede encontrar.

En uno de los primeros estudios centrados en analizar esta temática, Smith (1989), observó que no servía solo con hacer buenos planes de entrenamiento mental y con demostrar que éstos eran eficaces, sino que también las variables relativas al psicólogo del deporte que realiza la intervención, al deportista que está realizando el entrenamiento y al resto de agentes deportivos, eran relevantes a la hora de determinar los resultados finales de la intervención. Esto hizo que empezasen a proliferar las investigaciones relativas a los factores 
que inciden en el asesoramiento deportivo, demostrando que este proceso está también influido por las características del cliente, las del psicólogo deportivo, la naturaleza del problema, la calidad de la relación o las percepciones que tiene sobre el psicólogo del deporte y la psicología del deporte el propio deportista (Dorfman, 1990; Sexton y Whiston, 1994).

Ya en la pasada década, Harmison (2000), mencionaun estudio relevante en el que se pretende comprobar la influencia que tienen las percepciones de los deportistas sobre el psicólogo del deporte durante el aprendizaje de habilidades psicológicas, comparando los resultados de un programa de entrenamiento de habilidades psicológicas entre dos grupos de deportistas: un grupo que estaba convencido de su utilidad y otro grupo que no creía en la utilidad del entrenamiento. A pesar de que los dos grupos mejoraron, el grupo de deportistas que sí consideraba importante el entrenamiento en habilidades psicológicas obtuvo mejores resultados.

Revisando la bibliografía existente que analiza las expectativas sobre la efectividad del trabajo del psicólogo del deporte, así como los factores más relevantes para su inclusión, cabe destacar el trabajo de Silva (1984), en donde realiza una encuesta a entrenadores de escuelas superiores y universidades, relativa a la utilidad de los servicios de psicología del deporte. Los resultados de la encuesta mostraron dos realidades diferenciadas que había que tener muy en cuenta: por un lado, un porcentaje alto de entrenadores pensaban que era beneficioso trabajar con un psicólogo deportivo, o tener un equipo de asesoramiento psicológico; sin embargo, otros entrenadores lo veían más bien como un problema al considerar que ese trabajo entre el deportista y el psicólogo del deporte podía debilitar o influir negativamente en su relación con el deportista disminuyendo su autoridad, su liderazgo.

También Orlik y Partington (1987) realizaron un estudio al respecto. Entrevistaron a entrenadores olímpicos de Canadá concluyendo que uno de los mayores retos del psicólogo del deporte y de los servicios psicológicos en general eran las percepciones o connotaciones negativas que tenían los entrenadores con respecto a este servicio, ya que muchos de ellos consideran al psicólogo deportivo como un psicólogo clínico, es decir, como aquel que soluciona problemas clínicos de los deportistas y no como parte del equipo técnico que sirve para entrenar habilidades psicológicas. En este mismo estudio, entrevistaron a los 75 deportistas olímpicos canadienses que acudieron a los JJOO de 1984, encontrando que en 12 de los deportes representados, se había trabajado al menos con un psicólogo del deporte para preparar los Juegos, mostrando elevados niveles de satisfacción y de insatisfacción con el resultado del trabajo, lo que permitió establecer un perfil con las características que debían tener los consultores/psicólogos deportivos, para ser bien valorados por los deportistas.

Otro estudio realizado mediante encuestas se realizó en 1991 por Sullivan y Hodge. En este estudio se entrevistaron a
46 entrenadores nacionales y 68 deportistas de élite de Nueva Zelanda. La mayoría de los deportistas consideraron muy importante el entrenamiento psicológico e indicaron su interés por tener dentro de su equipo un psicólogo o servicio de psicología deportiva. Respecto a los entrenadores, un 94.9\% consideraban que el rendimiento de sus atletas era mayor si se llevaba a cabo un entrenamiento de habilidades psicológicas.

Respecto a los estudios realizados a partir del segundo milenio, destacan los trabajos del grupo de investigación de Martin. En el primero de sus trabajos centrados en este ámbito (Martinet al.,2001); con una muestra de 111 deportistas y de 166 universitarios no deportistas, se confirma la estructura de tres factores encontrados mediante el empleo del Expectations About Sport Psychology Consulting: compromiso personal, condiciones facilitadoras para el entrenamiento mental y experiencia del psicólogo del deporte. Respecto a las diferencias encontradas, éstas apuntan básicamente a discrepancias de género: mientras que las mujeres tienen unas expectativas más altas sobre la importancia del compromiso personal en la obtención de resultados, los hombres perciben los psicólogos deportivos son capaces de resolver los problemas de una manera más rápida. En un trabajo posterior (Martin, Kellman, Lavalle y Page, 2002); se crea el Sport Psychology Attitudes Revised; y en otra investigación posterior (Martin, 2005) analiza una muestra de 362 estudiantes de secundaria, y de 431 deportistas universitarios en Estados Unidos, encontrando que los deportistas masculinos y más jóvenes que realizaban deportes que implicaban contacto físico, tenían un estigma hacia la búsqueda de consultoría en psicología del deporte, planteando que los profesionales de esta área, deben ser sensibles a las características personales, a la experiencia y a las actitudes existentes hacia el rol del psicólogo del deporte.

Zakrajsek y Zizzi (2007, 2008), considerando las limitaciones que tenían el tipo de estudios que se habían realizado con respecto al tema de ideas previas de los entrenadores sobre el psicólogo del deporte y los servicios deportivos, en su mayoría cualitativos o de encuestas, adaptaron el cuestionario Sport Psychology Attitude-Revised (SPA-R) para entrenadores. La investigación se realizó con 113 entrenadores de natación, construyendo un cuestionario dirigido a saber cuál era la actitud de los entrenadores hacia el psicólogo del deporte. El cuestionario inicial constaba de 25 ítems divididos en cuatro factores: tolerancia del estigma, confianza en el psicólogo del deporte, apertura personal y preferencia cultural. Después de realizar el análisis exploratorio, el factor de preferencia cultural fue eliminado, quedando finalmente con tres factores, tolerancia del estigma, confianza en el psicólogo del deporte y apertura personal explicando un 40 por ciento de la varianza.

En otro interesante estudio, realizado mediante metodología cualitativa acerca de las ideas que tienen los entrenadores portugueses de fútbol profesional, sobre la labor del psicólogo 
del deporte (Neves, Garganta, Fernandes, Teixeira y Ferreira, 2011), se destaca también la importancia concedida a los aspectos psicológicos en el rendimiento, si bien no se profundiza, a nivel metodológico, en las causas que podrían estar explicando la no inclusión del psicólogo del deporte dentro del cuerpo técnico.

Respecto a los estudios publicados en castellano, Olmedilla, García y Garcés de los Fayos (1998), construyeron un cuestionario con el que pretendían obtener información relativa a la percepción de entrenadores de fútbol, en este caso de la región de Murcia (España), sobre el trabajo profesional de psicólogo del deporte. Este cuestionario pretendía obtener información sobre cinco factores: variables personales del entrenador, experiencia como futbolista y/ o entrenador, formación académica, variables técnicas y habilidades psicológicas y aspectos relacionados con lesiones deportivas. A partir de este estudio, se plantea la realización de una investigación que permita actualizar los resultados previos presentados, de manera que se pueda obtener un panorama actual sobre la percepción que los entrenadores de fútbol tienen sobre la figura del psicólogo del deporte. Para ello, se parte de los resultados presentados en el estudio de estas variables (De la Vega, García y Yagüe, 2003), lo que permite el análisis de los cambios que se han podido producir sobre las percepciones de los entrenadores acerca de la Psicología del Deporte a lo largo de 8 ańos, estableciendo las oportunas conclusiones que permitan, a su vez, futuros estudios que puedan comparar otros resultados con muestras recogidas en los próximos años. Se ha seleccionado este deporte, por su gran repercusión y por la facilidad de acceso a la muestra, lo que presenta un claro aporte a nivel aplicado respecto al análisis de variables que faciliten la inserción social y profesional del psicólogo del deporte.

Partiendo de este análisis del marco conceptual de partida, los objetivos del presente trabajo fueron: (1) analizar las ideas previas de los entrenadores de fútbol sobre la importancia de las variables psicológicas en el rendimiento, (2) analizar los posibles cambios en las ideas previas producidas en los entrenadores de fútbol entre 2003 y 2011, acerca de diversos aspectos relevantes de la psicología del deporte en el fútbol.

\section{Método}

\section{Diseño y Participantes}

La metodología utilizada ha sido propia de un diseño de encuesta o cuestionario ya que una de los objetivos principales era conocer la opinión o la percepción de los entrenadores de fútbol hacía la psicología del deporte, comparando los resultados de dos muestras distintas entre 2003 y 2011.

El estudio se realizó con dos muestras de participantes que cursaban el nivel I del curso de entrenador de fútbol de la
Escuela Madrileña de Entrenadores de Fútbol dependiente de la Escuela Nacional de Entrenadores de Fútbol. La primera de ellas recogida en el ańo 2003, estuvo compuesta por 103 participantes, con edades comprendidas entre los 22 y los 52 años $(M=29.91 ; S D=6.32)$, de los cuales un $68 \%$ habían sido entrenadores o monitores y el $52 \%$ jugadores. La segunda muestra fue recogida en el año 2011, compuesta por 95 participantes, con edades comprendidas entre los 17 y los 47 años $(M=29.30 ; S D=7.48)$, de los cuales el $57.9 \%$ habían sido entrenadores, el $45.3 \%$ jugadores y el $1.1 \%$ árbitros.

\section{Instrumentos}

El instrumento que se utilizó fue el Cuestionario de Evaluación de las Ideas Previas de los Entrenadores de Fútbol (CEIPEF, Anexo I). El cuestionario es totalmente anónimo y consta de cuatro partes diferenciadas: (a) información general del entrenador, (b) dos preguntas relacionadas con la importancia del psicólogo del deporte y su inclusión en el cuerpo técnico, puntuadas en una escala de 1 a 10, 1 siendo nada importante y 10 muy importante, (c) una parte abierta en el que se pregunta sobre la formación y los objetivos que consideran que debe pretender un psicólogo del deporte, (d) una última parte novedosa dentro de los cuestionarios realizados hasta la fecha, en el que se le pide a los entrenadores que numeren por orden de importancia a los siguientes componentes del equipo técnico si tuvieran que contar con ellos dentro de su cuerpo técnico y se contaran con recursos económicos limitados: preparador físico, psicólogo deportivo, fisioterapeuta, segundo entrenador, masajista y médico. Con este último ítem se pretende evaluar el valor atribuido al psicólogo del deporte cuando hay conflictos de intereses económicos a la hora de contratar profesionales, respecto a cuando no hay este conflicto. Después de la toma de datos de 2003, se hicieron dos pequeñas modificaciones en los datos sociodemográficos recogidos en el cuestionario: se incluyó una pregunta sobre los años de experiencia como entrenador, de manera que se pudiera analizar si esta variable tiene influencia sobre las percepciones acerca de la psicología del deporte; y se pasó a formato cerrado una de las preguntas abiertas sobre la formación que se piensa que debería tener el psicólogo del deporte, así como el modo de trabajo que debería desarrollar (aislado con los jugadores, en el campo, asesorando al entrenador y/o trabajando con el equipo en momentos puntuales), para facilitar su posterior análisis e interpretación (Anexo II).

\section{Procedimiento}

Para la realización del trabajo se contó con la aprobación de la Escuela Madrileña de Entrenadores de Fútbol, mediante la aceptación del consentimiento informado de participación en la investigación por parte de cada uno de los técnicos. Una 
vez recogidos los datos, fueron procesados y analizados mediante el programa estadístico SPSS 17.

El procedimiento seguido en ambos casos fue el mismo, respetando las normas relativas a la confidencialidad de los datos en ambos momentos temporales.

\section{Resultados}

Se han dividido los resultados del estudio en dos bloques. Un primer bloque donde se exponen los resultados de las pre- guntas comunes de la encuesta en los dos años y un segundo bloque donde se presentan algunos datos descriptivos de las nuevas preguntas introducidas en el año 2011.

\section{Variables comunes de 2003 y 2011.}

La tabla 1 muestra los estadísticos descriptivos de las preguntas comunes de los años 2003 y 2011.

Tabla 1. Estadísticos descriptivos de las variables comunes en 2003 y 2011.

\begin{tabular}{|c|c|c|c|c|}
\hline & \multicolumn{2}{|c|}{2003} & \multicolumn{2}{|c|}{2011} \\
\hline & M & $\mathrm{SD}$ & M & SD \\
\hline Importancia del PD en el fútbol (1-10) & 7.88 & 1.43 & 8.24 & 1.24 \\
\hline Contar con un PD dentro del cuerpo técnico (1-10) & 7.64 & 1.93 & 8.31 & 1.47 \\
\hline \multicolumn{5}{|l|}{$\begin{array}{l}\text { Orden de más a menos importancia del } 1 \text { al } 6 \text { (1=más } \\
\text { importante/ } 6=\text { menos importante) }\end{array}$} \\
\hline Preparador físico & 1.84 & 1.43 & 1.88 & 1.08 \\
\hline Psicólogo del deporte & 3.85 & 1.30 & 3.62 & 1.24 \\
\hline Fisioterapeuta & 3.18 & 1.16 & 3.57 & 1.35 \\
\hline Segundo entrenador & 3.75 & 1.95 & 2.59 & 1.72 \\
\hline Masajista & 4.85 & 1.23 & 5.28 & 1.09 \\
\hline Médico & 3.09 & 1.39 & 4.09 & 1.91 \\
\hline
\end{tabular}

Para comprobar si había diferencias entre las variables "Importancia del psicólogo del deporte en el fútbol" e "Importancia de contar con un psicólogo del deporte dentro del cuerpo técnico" en 2003 y 2011 se realizó un ANOVA de un factor, encontrándose diferencias significativas en la variable psicólogo del deporte dentro del cuerpo técnico $(F=6.48$; sig=0.01), pero no en la variable importancia del Psicólogo del deporte en el fútbol $(F=2.92 ;$ sig=0.09). Además, mediante un ANOVA de dos factores de medidas repetidas en un factor, se comprobó si las diferencias de medias en las puntuaciones entre los componentes del cuerpo técnico eran significativas entre si y entre los años 2003 y 2011. Ya que la muestra utilizada no cumplía los criterios de esfericidad (W de Mauchly= 0.679; sig=0.000) ni de igualdad de varianzas, se utilizaron estadísticos multivariados. Todos los estadísticos multivariados coinciden (con sig $=0,000$ ) en que hay diferencias significativas entre las posiciones de las diferentes figuras del cuerpo técnico y en la diferencia significativa de las posiciones, es decir, entre la interacción del factor cargo en el cuerpo técnico y los años.

En la tabla 2 se muestra la información de los efectos simples del factor "grupo" (2003 ó 2011) dentro de cada nivel del factor "cargo" (rol profesional ejercido). Los resultados indican que las puntuaciones acerca de la importancia de la figura del segundo entrenador, masajista y médico difieren entre los años 2003 y 2011, pero sin embargo no se producen cambios en las del preparador físico, psicólogo del deporte y fisioterapeuta. Este resultado, como se detallará en el apartado de conclusiones, debe ser valorado como de suma importancia para los objetivos del estudio, al no existir cambios significativos entre la percepción de los entrenadores evaluados en 2003 y en 2011 respecto a la importancia del psicólogo del deporte en el fútbol. Como el hecho de que existan diferencias en el factor "grupo" en los cargos segundo entrenador, masajista y médico, no significa que tengan puntuaciones diferentes con los cargos preparador físico, psicólogo del deporte y fisioterapeuta, se pasó a explorar esta posibilidad. 
Tabla 2. Diferencias de medias basadas en las medias marginales entre los diferentes roles profesionales en 2003 y 2011, ajustadas por Bonferroni

\begin{tabular}{lcccc}
\hline Figura & $(\mathrm{I})$ & $(\mathrm{J})$ & Diferencia de medias (I-J) & Sig.a \\
\hline \multirow{2}{*}{ Preparador Físico } & 2003 & 2011 & $0-033$ & 0.870 \\
\multirow{3}{*}{ Psicólogo del deporte } & 2011 & 2003 & -0.033 & 0.870 \\
& 2003 & 2011 & 0.319 & 0.115 \\
Fisioterapeuta & 2011 & 2003 & -0.319 & 0.115 \\
& 2003 & 2011 & -0.349 & 0.079 \\
Segundo entrenador & 2011 & 2003 & 0.349 & 0.079 \\
& 2003 & 2011 & 1.232 & 0.000 \\
Masajista & 2011 & 2003 & -1.232 & 0.000 \\
\multirow{3}{*}{ Médico } & 2003 & 2011 & -0.409 & 0.028 \\
& 2011 & 2003 & 0.409 & 0.028 \\
& 2003 & 2011 & -1.017 & 0.000 \\
& 2011 & 2003 & 1.017 & 0.000 \\
\hline
\end{tabular}

Por otra parte, en la tabla 3 se presentan los resultados respecto a la valoración sobre qué roles profesionales son los más importantes, desde el punto de vista de los entrenadores, si tuvieran que ajustar el presupuesto con el que cuentan para contratar y formar sus cuerpos técnicos, comparando también los resultados encontrados entre 2003 y 2011. Debido al incumplimiento de los supuestos exigibles, se ha realizado mediante comparaciones por pares con la Prueba T para muestras relacionadas, ajustando la tasa de error mediante el método Dumm-Bonferroni $(\alpha c)$. Cotejando estas diferencias de medias con las medias de la tabla 1 , se aprecia como las posiciones de los roles profesionales han variado de 2003 a 2011 de la siguiente manera: en el 2003 los entrenadores preferían contar, en primer lugar, con el preparador físico, en segundo término, con el fisioterapeuta y el médico, en tercera posición, el segundo entrenador y el psicólogo del deporte y, por último, el masajista. Sin embargo, en el año 2011, en primer lugar se mantiene el preparador físico, en segundo lugar asciende el segundo entrenador, en tercer lugar queda el psicólogo del deporte y el fisioterapeuta, relegando al médico al cuarto lugar y, a la última plaza, al masajista.

Tabla 3. Prueba T para muestras relacionadas corregidas con Dumm-Bonferroni.

\begin{tabular}{llccc|ccc}
\hline & & \multicolumn{3}{c}{2003} & \multicolumn{2}{c}{2011} \\
\cline { 3 - 7 } & & $\mathrm{T}$ & $\mathrm{gl}$ & Sig. & $\mathrm{T}$ & $\mathrm{gl}$ & Sig \\
\hline Preparador físico & Psicólogo del deporte & -9.08 & 88 & 0.000 & -8.81 & 84 & 0.000 \\
& Fisioterapeuta & -6.75 & 89 & 0.000 & -8.11 & 83 & 0.000 \\
& Segundo entrenador & -6.67 & 83 & 0.000 & -2.96 & 84 & 0.004 \\
& Masajista & -12.49 & 83 & 0.000 & -19.53 & 84 & 0.000 \\
& Médico & -5.34 & 87 & 0.000 & -11.56 & 84 & 0.000 \\
\hline Psicólogo del & Fisioterapeuta & 3.28 & 91 & 0.001 & 0.21 & 83 & 0.832 \\
deporte & Segundo entrenador & 0.51 & 83 & 0.612 & 4.36 & 84 & 0.000 \\
& Masajista & -4.67 & 86 & 0.000 & -8.02 & 84 & 0.000 \\
& Médico & 4.08 & 88 & 0.000 & -2.57 & 84 & 0.012 \\
\hline \multirow{3}{*}{ Fisioterapeuta } & Segundo entrenador & -1.95 & 84 & 0.054 & 3.65 & 83 & 0.000 \\
& Masajista & -10.09 & 86 & 0.000 & -9.71 & 83 & 0.000 \\
& Médico & 0.562 & 89 & 0.575 & -2.65 & 83 & 0.009 \\
\hline \multirow{2}{*}{ Segundo entrenadoryyyyyyyyyyyyy} & Masajista & -3.78 & 81 & 0.000 & -10.24 & 84 & 0.000 \\
& Médico & 2.05 & 82 & 0.430 & -5.80 & 84 & 0.000 \\
\hline Masajista & Médico & 8.17 & 85 & 0.000 & 5.207 & 84 & 0.000 \\
\hline
\end{tabular}


También se analizaron las relaciones entre las variables edad, formación académica, importancia del psicólogo del deporte en el fútbol, importancia del psicólogo del deporte en el cuerpo técnico y la posición en importancia del psicólogo del deporte. En este sentido, cabe destacar que la correlación significativa más elevada (.72), se obtiene en la muestra de 2011 en el sentido de que, cuanta más importancia se concede a la psicología del deporte, más se valora la posibilidad de contar con un psicólogo del deporte dentro del cuerpo técnico. Este resultado debería enfatizar la importancia de la formación ofrecida a los técnicos sobre la influencia de las variables psicológicas en el rendimiento, como posteriormente se discutirá.

Respecto a la formación que los técnicos consideran que debe poseer un psicólogo del deporte para que pueda trabajar en el fútbol, y respecto al modo en el que debería desarrollar su labor dentro del equipo, en la Tabla 4 se presentan los estadísticos descriptivos y resultados de las nuevas variables que se introdujeron para el cuestionario de 2011.

Tabla 4. Datos descriptivos de las nuevas preguntas introducidas en 2011: Formación preferida para un psicólogo del deporte en fútbol, y preferencia sobre su modo de trabajo.

\begin{tabular}{|c|c|c|}
\hline & \multicolumn{2}{|c|}{2011} \\
\hline & M & SD \\
\hline Años de experiencia entrenando & 2.84 & 2.68 \\
\hline Formación preferida para un psicólogo del deporte (1-10) & $(1-10)$ & $(1-10)$ \\
\hline 1. $C C A F D$ & 6.49 & 2.16 \\
\hline 2. Psicología & 8.64 & 1.93 \\
\hline 3. Máster en Psicología del Deporte & 8.26 & 1,93 \\
\hline 4. Entrenador nivel I & 6.12 & 2.31 \\
\hline 5. Entrenador nivel II & 5.8 & 2.34 \\
\hline 6. Entrenador nivel III & 6.05 & 2.66 \\
\hline Preferencia sobre el modo de trabajo del psicólogo del deporte (\%) & $\%$ & $\%$ \\
\hline Aislado con el deportista & 26.67 & 11.92 \\
\hline Asesoramiento personal al entrenador & 25.92 & 11.75 \\
\hline Entrenamiento en el campo & 23.26 & 12.59 \\
\hline Trabajo en momentos puntuales & 23.60 & 12.01 \\
\hline
\end{tabular}

Para ver si había diferencias significativas entre las diferentes respuestas de las preguntas, en este caso 3 y 6 , del cuestionario, se realizó una prueba $\mathrm{T}$ de Student para muestras relacionadas con la tasa de corrección de Dumm-Bonferroni. En la pregunta 3, relativa a la formación del psicólogo deportivo, hay diferencias entre todas las respuestas menos en la valoración entre la formación en Ciencias de la Actividad Física y Deporte (CCAFD) y entrenador nivel I $(t=1.057$; $p=0.294)$, formación en CCAFD y entrenador nivel III ( $t=$ 1.623; $p=0.109)$, entre psicólogo y máster en Psicología del Deporte $(t=1.352 ; p=0.181)$ y entre los diferentes niveles de curso de entrenador, I y el II $(t=1.276 ; p=0.206)$ y el II con el $\operatorname{III}(t=0.242 ; p=0.809)$. En la pregunta 6 , que hace referencia la distribución del $100 \%$ de la importancia entre diferentes metodologías usadas por el psicólogo del deporte, no se encontraron diferencias significativas entre las variables. Cabe destacar que no se encontraron relaciones significativas con los ańos de experiencia y el resto de variables.

\section{Discusión}

En el presente trabajo se estudiaron las ideas previas de futuros entrenadores de fútbol hacía la Psicología de la Actividad Física y el Deporte, así como el papel del psicólogo dentro del equipo deportivo.

Los resultados obtenidos sobre la importancia que conceden los futuros entrenadores a la psicología del deporte dentro de su ámbito aplicado son altos, ya que la media de las puntuaciones que se han obtenido en esta pregunta han sido de 7.88 y 8.24 en las muestras de 2003 y 2011 respectivamente, siguiendo la línea de otros estudios similares en este ámbito (Olmedilla et al., 1998). Respecto a lo positivo de la inclusión del psicólogo del deporte dentro del cuerpo técnico también se encuentran medias elevadas en ambos años, concretamente de 7.64 y 8.31 respectivamente. Cabe destacar que, aunque en la variable "importancia del psicólogo del deporte en el fútbol" no se han encontrado diferencias significativas en las puntuaciones de 2003 y 2011, en la variable "psicólogo del deporte dentro del cuerpo técnico" sí se han encontrado. Es 
decir, que se ha modificado en sentido positivo la percepción de la necesidad de incluir un psicólogo del deporte dentro del cuerpo técnico de un equipo de fútbol.

Pero en este estudio no solo se pretendía obtener datos sobre la percepción autoinformada sobre el ámbito de la Psicología del Deporte y sobre el papel del psicólogo deportivo, sino que uno de nuestros intereses se centraba en conocer si, pese a destacar la posible relevancia de los factores psicológicos en el rendimiento deportivo, e incluso del rol que puede llegar a ejercer el psicólogo del deporte dentro del contexto futbolístico, en el momento de contratar a un profesional de la psicología del deporte, se prioriza antes a otras figuras que suelen integrar el cuerpo técnico. Si esta pregunta es importante en una situación socio-económica normal, aún lo es más en la coyuntura actual en la que se encuentra inmerso nuestro país, lo que provoca que el futuro laboral y profesional del sector de la psicología del deporte pueda verse afectado. En este sentido, de los seis posibles roles profesionales a elegir en orden jerárquico de importancia, (preparador físico, fisioterapeuta, segundo entrenador, médico, masajista y psicólogo del deporte), tanto en 2003 como en 2011, el psicólogo del deporte queda en tercera posición, no existiendo cambios significativos en el transcurso de 8 años.

Cabe destacar, aunque hay que tomarlo con precaución, que se han encontrado correlaciones negativas significativas, aunque muy bajas, entre la importancia de la psicología del deporte y la importancia de que esté un psicólogo del deporte en el cuerpo técnico con la prioridad en la contratación, es decir, cuanto más importante perciben al psicólogo deportivo, mayor prioridad le dan a la hora de seleccionar a miembros de su cuerpo técnico. Este dato resulta especialmente alentador y presenta el reto de continuar con la formación adecuada en psicología del deporte, no sólo en los cursos de entrenadores de fútbol, sino también en general en los cursos de técnicos deportivos de cualquier modalidad. Se debe recordar que en nuestro país, a diferencia de otros, la formación académica en Ciencias de la Actividad Física y del Deporte, en donde por supuesto que en los planes de estudio se desarrollan las materias de "Psicología de la Actividad Física y del Deporte", no implica que los futuros graduados obtengan titulaciones de técnicos deportivos. Para ser técnico en deportes específicos, la persona interesada puede optar por cursos académicos en instituciones privadas o puede optar por la vía también privada de las federaciones deportivas, lo que implica la necesidad de hacer estudios que puedan analizar con un nivel elevado de profundidad la realidad académica que, en lo relativo a la psicología del deporte, existe en la formación de técnicos deportivos.

De hecho, mucho nos tememos que no se cumplen, en el caso de un elevado porcentaje de federaciones, con las horas marcadas por decreto ley en las materias que, como la Psicología del Deporte, forman parte del denominado "Bloque común”, lo que podría ser estudiado, desde nuestra perspectiva, en función de dos hipótesis esenciales: a las federaciones minoritarias las cuesta mucho organizar cursos de técnicos deportivos porque muchas de ellas no tienen un número suficiente de alumnos interesados, por lo que los costes de organización se disparan y no hay dinero suficiente para costear el volumen de horas que se deben impartir y, como hipótesis complementaria, la escasa importancia otorgada a las materias que componen el bloque común, respecto al bloque especifico, provoca que la formación sea deficitaria en las primeras.

Con respecto a las variables de información general del entrenador, la que ha resultado tener una relación significativa, aunque nuevamente muy baja, con las variables relativas a la importancia percibida ha sido la edad tanto en 2003 como en 2011 (Tablas 3 y 4), es decir, a mayor edad más importancia del psicólogo del deporte en el fútbol, pero esta correlación significativa no se mantiene cuando tiene que priorizar sobre otros componentes del equipo técnico o cuando se habla del psicólogo del deporte en el cuerpo técnico. Ni la formación, ni la experiencia han dado resultados significativos con el resto de variables.

De las preguntas nuevas añadidas en 2011 cabe destacar que los entrenadores consideran prioritaria la formación como psicólogo y máster en psicología deportiva para un psicólogo del deporte, quedando en un segundo plano CCAFD y los cursos de entrenador. Además, sobre las metodologías que tendría que utilizar un psicólogo del deporte consideran las cuatro propuestas igual de relevantes, tanto el trabajo con el deportista, el trabajo en el campo, con el entrenador y momentos puntuales con el equipo. Esto nos indica que habría que seguir profundizando en las habilidades del psicólogo del deporte en el trabajo de campo y en contextos situacionales puntuales, que son los menos desarrollados en los programas de formación de manera específica.

En resumen, los futuros entrenadores de fútbol consideran relevante la psicología del deporte tanto como disciplina como la figura del especialista dentro del cuerpo técnico. Pero no se debe perder de vista uno de los mayores problemas del autoinforme, y es que muchas veces por simple deseabilidad las respuestas pueden estar sesgadas (incluso aunque el cuestionario sea anónimo). Por este motivo consideramos fundamental seguir investigando este tipo de cuestiones en la línea de los ítems en los que se obligue a la gente a elegir o con pruebas en las que el objetivo no sea tan evidente, para evitar este tipo de sesgos. Puesto que lo que realmente nos interesa de las percepciones es el comportamiento que realizan las personas por las mismas.

\section{Aplicaciones Prácticas}

Tal y como se ha puesto de manifiesto a lo largo del trabajo de investigación, un elemento crucial en el desempeño profesio- 
nal del psicólogo del deporte, se centra en conocer cómo nos perciben los profesionales que son en gran parte responsables de nuestra contratación: los entrenadores. En su aplicación al fútbol, resulta especialmente relevante que el profesional que se quiere introducir, a nivel profesional, en este deporte, sepa elementos como, por ejemplo, que se prefiere trabajar con psicólogos con un Máster de especialización en Psicología del Deporte, que también se valoran los conocimientos específicos del fútbol, así como que se considera relevante el trabajo no sólo independiente con el jugador, sino también de asesoramiento al técnico (counseling), y el trabajo aplicado al propio campo de entrenamiento.
El obtener, en los datos recogidos en el 2011, un tercer puesto en la preferencia de los profesionales con los que contaría un entrenador, nos permite presentar un futuro profesional esperanzador, en el que los futuros profesionales encuentran ya una buena predisposición de los técnicos para realizar su trabajo (labor conseguida a lo largo de más de dos décadas de trabajo de muchos especialistas en el área).

Agradecimientos: El presente estudio se ha desarrollado dentro del marco de dos proyectos de investigación del Plan Nacional I+D+I, del Ministerio de Ciencia e Innovación: Ref. DEP2011-27282; y Ref. PSI2011-27000.

\section{Referencias}

1. De la Vega, R., García, T. y Yagüe, J.M. (2003). Ideas previas de los entrenadores de fútbol sobre la noción de psicología "en el deporte". En Libro de Actas del II Congreso Internacional de Psicología Aplicada al Deporte, UNED, Madrid (pp.177). Madrid: Dykinson.

2. Dorfman, H.A (1990). Reflections on providing personal and performance enhancement consulting services in professional baseball.The Sport Psychologist, 4(4), 341-346.

3. Gucciardi, D.F. (2012). Measuring mental toughness in sport: a psychometric examination of the Psychological Performance Inventory-A and its predecessor. Journal of personality assessment, 94(4), 393-403. DOI: $10.1080 / 00223891.2012 .660292$

4. Harmison, R.J. (2000). Athletes attitudes toward seeking sport psychology consultation: development and validation of the sport psychology attitudes questionnaire.Tesis Doctoral. Univesity of North Texas.

5. Martin, S.B. (2005). High school and college athletes' attitudes toward sport psychology consulting.Journal of Applied Sport Psychology, 17, 127139.DOI: $10.1080 / 10413200590932434$

6. Martin, S. B., Akers, A., Jackson, A. W., Wrisberg, C. A., Nelson, L., y Leslie, P. J.. (2001). Male and female athletes' and nonathletes' expectations about sport psychology consulting.Journal of Applied Sport Psychology, 13, 18-39.

7. Martin, S. B., Kellmann, M., Lavallee, D., \& Page, S. J. (2002). Development and psychometric evaluation of the sport psychology attitudesRevised form: A multiple group investigation. The Sport Psychologist, 16, 272-290

8. Montero, I. y León, O.G. (2002). Clasificación y descripción de las metodologías de investigación en Psicología. Revista Internacional de Psicologia Clínica y de la Salud IInternational Journal of Clinical and HealthPsychology, 2, 503-508

9. Neves, A., Garganta, J.M., Fernandes, P., Teixeira, D.F. y Ferreira, A.M. (2011). Whatportuguese premier league coachesthinkabouttheimportance of thepsychologicalintervention in professional Soccer?. Motriz.
Journal of PhysicalEducation. UNESP, 17(1), 128-137.

10. Olmedilla, A., García, C. y Garcés de los Fayos, E. J. (1998). Un análisis del papel profesional del psicólogo del deporte desde la percepción del entrenador de fútbol. Revista de Psicología del Deporte, 13, 95-111.

11. Orlick, T. yPartington, J. (1987). The sport psychology consultant: Analysis of critical components as viewed by Canadian Olympic athletes. The Sport Psychologist, 1, 14-17.

12. Sexton, T. L., yWhiston, S. C. (1994). The status of the counseling relationship: An empirical review, theoretical implications, and research directions. The Counseling Psychologist, 22, 6-78.

13. Silva, J. (1984). The status of sport psychology: A national survey of coaches. Journal of Physical Education, Recreation, and Dance, 55(7), 46-49.

14. Smith, R.E. (1989). Applied sport psychology in the age of accountability. Journal of Applied Sport Psychology, 1, 166-180.

15. Sousa, C., Cruz, J., Torregrosa, M., Vilches, D. yViladrich, C. (2006) Evaluación conductual y programa de asesoramiento personalizado a entrenadores (PAPE) de 119 deportistas jóvenes. Revista de Psicología del Deporte, 15 (2), 263-278.

16. Sullivan, J., y Hodge, K. P. (1991). A survey of coaches and athletes about sport psychology in New Zealand. The Sport Psychologist, 5, 140151

17. Zakrajsek, R., yZizzi, S. (2007). Factors influencing track and swimming coaches intentions to use sport psychology services. Athletic Insight: Online Journal of Sport Psychology, 9(2), Retrieved January 26, 2013 from http://www.athleticinsight.com/Vol9Iss2/CoachesIntentions.htm.

18. Zakrajsek, R. yZizzi, S. (2008). How do coaches' attitudes change when exposed to a sport psychology workshop? Journal of Coaching Education, Vol 1(1).Retrieved January 26, 2013 from http://www.aahperd.org/naspe/jce/pdf_files/Zakrajsek_Zizzi_JCE_2008.pdf

\section{Anexo I}

Edad: $\quad$ Fecha: Sede:

Formación deportiva:

Formación académica: 
Instrucciones: A continuación se te presentan una serie de cuestiones sobre la Psicología del Deporte. Este cuestionario es completamente anónimo y confidencial, por lo que te solicitamos que respondas con la mayor sinceridad posible. En las preguntas primera y segunda, rodea con un círculo la puntuación que desees señalar.

1. ¿Qué importancia concedes a la psicología del deporte como área aplicada en el fútbol?

Mínima 1-2-3-4-5-6-7-8-9-10 Máxima

Razona y justifica brevemente tu respuesta:

2. ¿Crees que es positivo contar con un psicólogo del deporte dentro del cuerpo técnico?.

Nada positivo 1-2-3-4-5-6-7-8-9-10 Muy positivo

Razona y justifica brevemente tu respuesta:

3. ¿Cuál es la formación que consideras que debe tener un psicólogo del deporte que trabaje en fútbol?.
4. Describe cuáles crees que son los 3 objetivos básicos en el trabajo de un PSICÓLOGO DEL DEPORTE.

1.

2.

3.

5. Describe cuáles crees que son los 3 objetivos básicos en el trabajo psicológico que debe realizar el ENTRENADOR: 1.

2.

3.

6. Establece un orden de prioridad desde el más importante (1), hasta el menos importante (6), en función de la importancia que tiene para ti contar con cada uno de los siguientes profesionales relacionados con tu deporte (si alguno de ellos consideras que no resulta pertinente en tu deporte, simplemente deja su espacio en blanco y numera de 1 a 5):

Preparador físico ( )

Psicólogo deportivo ( )

Fisioterapeuta ( )

Segundo entrenador ( )

Masajista ( )

Médico ( )

\section{Anexo II}

Edad: Fecha:

Curso de entrenadores de fútbol de nivel:

Formación deportiva:

Formación académica:

Años de experiencia como entrenador:

Instrucciones: A continuación se te presentan una serie de cuestiones sobre la Psicología del Deporte. Este cuestionario es completamente anónimo y confidencial, por lo que te solicitamos que respondas con la mayor sinceridad posible. En las preguntas primera y segunda, marca con una cruz la puntuación que desees señalar.

1. ¿Qué importancia concedes a la psicología del deporte como área aplicada en el fútbol?

\begin{tabular}{cccccccccc}
\hline $\begin{array}{c}\text { Mínima } \\
1\end{array}$ & 2 & 3 & 4 & 5 & 6 & 7 & 8 & 9 & 10 \\
\hline
\end{tabular}

Razona y justifica brevemente tu respuesta:
2. ¿Crees que es positivo contar con un psicólogo del deporte dentro del cuerpo técnico?

\begin{tabular}{|l|l|l|l|l|l|l|l|l|l|}
\hline $\begin{array}{l}\text { Nada positivo } \\
1\end{array}$ & 2 & 3 & 4 & 5 & 6 & 7 & 8 & 9 & \begin{tabular}{l} 
Muy positivo \\
\hline
\end{tabular} \\
\hline
\end{tabular}

Razona y justifica brevemente tu respuesta:

3. ¿Cuál es la formación que consideras que debe tener un psicólogo del deporte que trabaje en fútbol? Señala en una escala de 1 (Nada importante) a 10 (Muy importante) como de importante te parece la siguiente formación. 


\begin{tabular}{|c|c|c|c|c|c|c|c|c|c|c|}
\hline \multirow[t]{2}{*}{ Formación } & \multicolumn{10}{|c|}{ 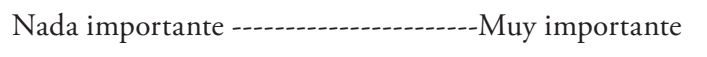 } \\
\hline & 1 & 2 & 3 & 4 & 5 & 6 & 7 & 8 & 9 & 10 \\
\hline \multicolumn{11}{|c|}{ Licenciado en Ciencias de la Actividad Física y el Deporte } \\
\hline \multicolumn{11}{|l|}{ Licenciado en Psicología } \\
\hline \multicolumn{11}{|l|}{ Máster en Psicología del Deporte } \\
\hline \multicolumn{11}{|l|}{ Entrenador de Fútbol de Nivel I } \\
\hline \multicolumn{11}{|l|}{ Entrenador de Fútbol de Nivel II } \\
\hline Entrenador de Fútbol de Nivel III & & & & & & & & & & \\
\hline
\end{tabular}

En el caso de considere que resulta relevante alguna otra formación para el psicólogo del deporte, señálela en la siguiente tabla (Indica cuál y puntúela nuevamente de 1 a 10):

\begin{tabular}{|c|c|c|c|c|c|c|c|c|}
\hline \multirow[t]{2}{*}{ Formación } & \multicolumn{8}{|c|}{ 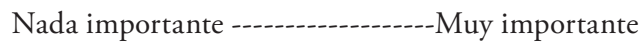 } \\
\hline & 1 & 3 & 4 & 5 & 6 & 7 & 8 & 10 \\
\hline
\end{tabular}

4. Describe cuáles crees que son los 3 objetivos básicos en el trabajo de un PSICÓLOGO DEL DEPORTE.

1.

2.

3.

5. Describe cuáles crees que son los 3 objetivos básicos en el trabajo psicológico que debe realizar el ENTRENADOR:

1.

2.

3.
6. Distribuye un $100 \%$ de importancia entre las siguientes metodologías de trabajo que puede realizar un psicólogo del deporte en el fútbol:

a. Trabajo aislado con el deportista. ( \%)

b. Asesoramiento personal al entrenador. ( \%)

c. Entrenamiento psicológico aplicado al campo y realizado por el propio psicólogo. ( \%)

d. Trabajo con el equipo realizado en momentos puntuales. $(\%)$

7. Establece un orden de prioridad desde el más importante (1), hasta el menos importante (6), en función de la importancia que tiene para ti contar con cada uno de los siguientes profesionales relacionados con tu deporte (si alguno de ellos consideras que no resulta pertinente en tu deporte, simplemente deja su espacio en blanco y numera de 1 a 5):

Preparador físico ( )

Psicólogo deportivo ( )

Fisioterapeuta ( )

Segundo entrenador ( )

Masajista ( )

Médico ( ) 